\title{
MUJERES RURALES EN EL SOSTENIMIENTO DE LA SOBERANÍA ALIMENTARIA Y LOS EQUÍVOCOS PATRIARCALES EN LOS ESTUDIOS ETNOBIOLÓGICOS DEL NORTE DE LA PATAGONIA
}

\author{
RURAL WOMIEN IN SUSTAINING FOOD SOVEREIGNTY AND \\ PATRIARCHAL MISUNDERSTANDINGS IN ETHNOBIOLOGICAL STUDIES \\ IN NORTHERN PATAGONIA
}

\author{
Ana Haydeé $\mathrm{LADIO}^{1}$ * \\ ${ }^{1}$ INIBIOMA (Instituto de investigaciones en Biodiversidad y Medio Ambiente, CONICET-UNComahue). Quintral 1250. 8400. S.C. de \\ Bariloche. Argentina*ahladio@gmail.com
}

Submitted: 05/11/2020; Accepted: 21/01/2021; Published: 19/02/2021

\section{RESUMEN}

Desde una aproximación etnográfica cualitativa re-examiné mis trabajos publicados e hice un repaso de los resultados y sus discursivas (éticas y émicas) en el tratamiento del rol de las mujeres en la vida rural, distinguiendo aquellos abordajes acordes con la perspectiva de género, así como también mis equívocos patriarcales. Estos son los desentendimientos producidos por el sesgo patriarcal que impidieron visibilizar a las mujeres en forma completa. La revisión se basó en estudios realizados en comunidades rurales minifundistas del Norte de la Patagonia (Argentina), principalmente de origen Mapuche y/o Mapuche-Tehuelche y que han abordado distintos temas vinculados a la soberanía alimentaria. La fuerte construcción social existente acerca de las mujeres "cuidadoras" ha atravesado todos los trabajos, hecho que hace necesario repesar esa construcción de sentido y reflexionar junto con las mujeres rurales y las investigadoras e investigadores. La invisibilización de mujeres y hombres, la sobre-generalización y la simplificación de resultados son equívocos que pueden servir de enseñanza para tener una mirada de género más sensible en el futuro.

PALABRAS ClAVE: Agricultura familiar, Ética del cuidado, Sesgos de género, Mapuche, Tehuelche.

\begin{abstract}
From a qualitative ethnographic approach, I re-examined my published works. I have reviewed the results and their discursive (ethical and emic) in the treatment of the role of women in rural life, distinguishing those approaches that are consistent with the gender perspective, as well as my patriarchal misunderstandings. These misunderstandings are produced by the patriarchal bias that prevented women from being fully visible. The review was based on studies carried out in rural smallholder communities in Northern Patagonia (Argentina), mainly of Mapuche and/or MapucheTehuelche origin, which have addressed different aspects related to food sovereignty. The strong social construction that exists regarding women "caretakers" has permeated all $\mathrm{f}$ the work, making it necessary to review this construction of meaning and to reflect on it together with rural women and researchers. The invisibility of women and men, over-generalization and simplification of results are misconceptions that can serve as lessons for a more gender-sensitive view in the future.
\end{abstract}

KEYWORDS: Family farming, Ethics of care, Gender bias, Mapuche, Tehuelche. 


\section{INTRODUCCIÓN}

La etnobiología es una disciplina que proyecta procesos de cambio en sus evidencias, en sus marcos conceptuales y en sus aproximaciones (BALDAUF, 2019). La capacidad auto reflexiva es indispensable para repensarnos en nuestro rol en la sociedad, que desde mi punto de vista, es de ser agentes de interlocución entre el sistema de conocimientos científicos y el sistema de conocimientos locales para un diálogo equitativo de saberes (LADIO, 2017).

La inclusión de la perspectiva de género es uno de los cambios más sustanciales de estos últimos tiempos en nuestra disciplina (VIEIRA y MILWARD-de-AZEVEDO, 2018). Sin embargo, no parece ser un camino fácil, necesita de reflexiones, despejar resistencias y generar nuevos acuerdos. Todos los participantes de los estudios etnobiológicos (investigadores e integrantes de las comunidades locales), por medio de distintos procesos de socialización, iniciando en el seno familiar, incorporamos, somatizamos o interiorizamos fuertes estructuras sociales de pensamiento. Vivenciamos este proceso de estructuración social de manera espontánea y natural, casi sin darnos cuenta, lo que se conoce como el concepto de habitus (BOURDIEU, 2007). Esta noción de habitus nos permitiría reconocer la dificultad existente en desentrañar sesgos patriarcales que portamos de manera profunda. También, nos invita a revisar los cambios que dejan entrar luz en estos habitus, que son necesarios para redirigir nuestra mirada hacia el interior de la vida de las mujeres en un mundo machista.

Problematizar e interpelar las marcas de género y clase que portamos como etnobiólogas y etnobiólogos es muy necesario (sensu BROUCHOUD, 2017). Revisar textos, grabaciones y recuerdos de trabajos de campo anteriores con otras miradas y que nos permitan analizar las relaciones sociales que entablamos en el pasado, nos puede ubicar en un nuevo territorio de comprensión. La pregunta no es si tuvimos o no sesgos patriarcales en nuestras investigaciones, sino si tenemos la disposición para reconocer la parcialidad del punto de vista. Otra opción es negarnos a su re-examinación y evitar la autocrítica, aunque corremos el riesgo de no reflexionar sobre una visión del mundo que deja afuera sujetos o que convalida procesos de opresión o exclusión.

En un trabajo anterior (LADIO, 2020a), señalo que los trabajos etnobiológicos en áreas rurales poco han podido desentrañar jerarquías patriarcales que operan en los cotidianos de las sociedades de estudio, y que también persisten entre los propios investigadores e investigadoras. Los sesgos patriarcales implican convalidar la creencia que 
fomenta que las diferencias de orden biológico que existen entre mujeres y varones justifican desigualdades y sometimientos sobre las primeras. Estos esquemas de poder, dejan a las mujeres totalmente relegadas o auto-relegadas en innumerables espacios de decisión de la vida cotidiana (SILIPRANDI, 2010; GILLIGAN, 2013).

La histórica subordinación de las mujeres ante esquemas patriarcales es de larga data y se aplica a todas las sociedades urbanas y rurales de casi todo el mundo. Es decir, que los mismos sesgos actúan bidireccionalmente en una investigación etnobiológica donde hay un encuentro entre personas que pertenecen a distintos ámbitos y/o culturas. En particular, quiero detenerme en los trabajos vinculados a las áreas rurales, ligados a la soberanía alimentaria, en donde evidencio particularmente que las mujeres no han tenido el tratamiento adecuado en términos metodológicos y/o conceptuales para visibilizar su rol sustancial.

\section{LAS MUJERES RURALES Y LA SOBERANÍA ALIMENTARIA}

Según datos de la Organización de las Naciones Unidas para la Alimentación y la Agricultura (FAO, 2020), 1/3 de la población mundial corresponde a mujeres rurales, las cuales representan el $43 \%$ de su mano de obra. Sin embargo, las estadísticas muestran que ellas no acceden de igual manera que los hombres a los beneficios económicos de ese trabajo, ni a la propiedad de la tierra, ni a capacitación agrícola, ni créditos etc.

Las mujeres rurales destinan gran cantidad de horas al día en el trabajo doméstico, agrícola y reproductivo asignado en sus sociedades que está íntimamente ligado a la soberanía alimentaria. Desde la infancia, las mujeres y niñas van aprendiendo actividades diversas, a modo de habitus, como la crianza de niñas y niños, el cuidado de la salud familiar, la preparación de alimentos, las actividades agrícolas, el acarreo de agua y leña. Según datos de la FAO (2020), el 80\% de los hogares sin agua corriente depende del trabajo de mujeres y niñas para conseguir agua. Además, entre el 60 y $80 \%$ de la producción de alimentos en países en desarrollo está en manos de las mujeres rurales (VIZCARRA BORDI, 2008).

Se ha encontrado que, en muchas regiones rurales, las mujeres destinan en el trabajo doméstico 14 horas al día en promedio, de las cuales 5 horas corresponden a la recolección de leña, el acarreo del agua, recolección de plantas y hongos y el pastoreo de pequeños rebaños. Además, 4 horas en promedio se destinan para la preparación de alimentos. El resto del tiempo es distribuido en trabajos de "ayuda" a la agricultura de subsistencia, lavar 
ropa, cuidar a los menores y ancianos, preparar ceremonias comunitarias o familiares, entre otras actividades propias de cada región y cultura (VIZCARRA BORDI, 2008).

Trpin, Rodriguez y Brouchoud (2017) han denunciado que la lógica neoliberalpatriarcal que solo pondera el trabajo productivo rural que genera dinero, en general realizado por hombres, deja invisible el trabajo doméstico o el informal llevado a cabo principalmente por las mujeres. Por otra parte, si bien cada vez más mujeres participan en programas de desarrollo, actividades organizativas, venta de productos en ferias, que implican procesos de empoderamiento, para la mayoría, esto representa una mayor sobrecarga de responsabilidades dado que las actividades de cuidado doméstico no son equitativamente repartidas con los varones (VIZCARRA BORDI, 2008; PENA, 2017).

\section{EL ENFOQUE ETNOBIOLÓGICO EN LA SOBERANÍA ALIMENTARIA}

Según Vía Campesina (2018), la soberanía alimentaria es el derecho de cada pueblo a definir su alimentación y sus formas de obtener alimentos. Se vincula con el respeto a la cultura culinaria de cada comunidad y a que las sociedades tengan el control directo y democrático de su alimentación. No solo refiere a la alimentación del presente sino también a que se garantice su sostenibilidad en el tiempo para que las futuras generaciones puedan acceder a alimentos sanos y seguros acorde con sus tradiciones culinarias. La soberanía alimentaria se trata de un proceso en acción dado que muchos cambios deben ser realizados para que estos derechos sean garantizados. Es por ello que desde la misma Vía Campesina (2018), organización que creó dicho término, consideran que el enfoque de género no debe estar excluido.

Cabe aclarar que no se trata solo de alimentos, implica múltiples aspectos que la etnobiología ha estudiado, como la recolección del agua y la leña, la trasmisión de conocimientos y prácticas vinculadas con el cuidado del ambiente, la cría de animales de traspatio y/o a pequeños y grandes rebaños, la horticultura, entre tantos otros. Incluye tradiciones, pero también innovaciones para asegurar la sustentabilidad socioambiental de acuerdo con las pautas culturales de cada uno de los pueblos (VÍA CAMPESINA, 2018).

El rol que cotidianamente tienen las mujeres para asegurar una alimentación sana y segura para la familia y la comunidad ha sido extensamente señalado (TASSI; BEZERRA, 2020). Los estudios etnobiológicos han aportado en numerosos aspectos de la soberanía alimentaria, pero muchas veces se han conformado con diferenciar conocimientos y prácticas entre las mujeres y los varones (PFEIFFER; BUTZ, 2005). Poco hemos 
profundizado al interior de la vida de todas esas mujeres sobre las complejidades/desigualdades que no emergen sin una sólida perspectiva de género.

Un caso paradigmático que ilustra las parcialidades existentes en este tema es el trabajo de Bain (1993) en México que evidenció que las mujeres tienen un rol muy significativo en la producción de alimentos, resultados que nadie se animaría a cuestionar según los distintos estudios de caso existentes desde la etnobiología (DUARTE ALMADA; OLIVEIRA E SOUZA, 2017). Pero en este trabajo, también se detectó que a la hora de comer, los hombres comen primero que mujeres y niños, mostrando jerarquías de poder que podrían tener consecuencias nutricionales. Esta situación también se ha encontrado en estudios de América del Sur y Asia (BYERS; SAINJU, 1994; VIZCARRA BORDI, 2008).

Las mujeres son las gestoras, las que más saben sobre alimentos silvestres y cultivados, sin embargo como propuso Kelleher (1997) se evidencia un proceso de feminización del hambre (o masculinización de la riqueza), el cual cobra sentido para comprender la tensión que soportan las mujeres frente al hambre familiar, local y/o regional. Lamentablemente, las consecuencias de la pandemia COVID 19, como otras hambrunas anteriores, revelan que son las mujeres y las niñas las que más han padecido y están padeciendo el hambre y situaciones de vulnerabilidad en comparación con los hombres (VIZCARRA BORDI, 2008; FAO, 2020).

Entonces, se trata de revisar aquellas prácticas profesionales que llevaron a interpretaciones sesgadas y realidades fragmentadas que vienen perpetuándose en el tiempo como habitus (por ejemplo en trabajos publicados) y que reducen la complejidad del mundo de las mujeres. En este trabajo, reflexionaré sobre lo que llamo mis equívocos patriarcales, estos son los desentendimientos producidos por el sesgo patriarcal que impidieron visibilizar a las mujeres en forma completa, no como producto de una incompetencia, sino como resultante de la perpetuación automática de una estructura sociocultural (habitus). En este sentido, Eichler y Burke (2006), hablan del triángulo del sesgo, una trilogía que perpetúa sesgos patriarcales de la siguiente manera: 1) convalidando jerarquías existentes, 2) fallando en examinar las diferencias y/o 3) apelando a doble estándares. Evaluar estas alternativas nos permite corregir y repensar nuestras prácticas profesionales dado que nos invita a constatar si hemos tenido posicionamientos colonizados, convalidamos una posición dominante, fuimos insensibles, sobre-generalizamos, de-contextualizamos o asumimos una homogeneidad inexistente. 


\section{EL ROL DE LAS MUJERES EN LA PATAGONIA RURAL}

Desde una aproximación etnográfica y cualitativa realicé una examinación de un total de 55 trabajos publicados de mi autoría (2001-2020) sobre soberanía alimentaria. Se trata de estudios realizados en comunidades rurales minifundistas del Norte de la Patagonia (Argentina), principalmente de origen Mapuche y/o Mapuche-Tehuelche y que han abordado distintos temas vinculados a la temática (uso de plantas y animales comestibles y medicinales silvestres, ganadería criancera, uso de plantas leñateras, uso del agua, horticultura familiar y forestación peridoméstica).

No ha sido intención realizar una revisión positivista ni estandarizada en categorías, sino profundizar en la re-interpretación de los principios generadores y organizadores de prácticas y representaciones que subyacen en los textos. En un proceso recursivo y no lineal, pude hacer un repaso de las discursivas (éticas y émicas) en el tratamiento del rol de las mujeres en la vida rural, distinguiendo aquellos abordajes acordes con la perspectiva de género (LADIO, 2020a), así como también mis equívocos patriarcales.

Las comunidades rurales involucradas se caracterizan por vivir en condiciones de subsistencia, en territorios comunitarios. Son también quienes han sufrido terribles procesos de despojo desde la llegada del hombre blanco a la región (PEREZ, 2011). Sus modos de vida se vinculan directamente al uso de recursos naturales y son representativos de la vida rural patagónica.

\section{1 Las mujeres cuidadoras y trasmisoras}

En primer lugar, la re-interpretación de los trabajos de campo muestra que el valor que las mujeres rurales le dan a la soberanía alimentaria está directamente asociado con el rol asumido de "cuidadoras". Desde ese lugar simbólico se insertan en la estructura productiva y familiar, pero también en su manera de comprender a la Naturaleza, la crianza de sus seres y sus ciclos. Dicha responsabilidad, fuertemente designada, no parece acontecer entre los hombres, aunque ello no implique que no sientan un gran compromiso en las decisiones vinculadas con la gestión pecuaria ni el cuidado ambiental, especialmente evidente en el caso de los varones de las comunidades Mapuche y Tehuelche (LADIO, 2020b). Pero, aspectos directamente vinculados con la alimentación y salud familiar, la horticultura y el cuidado de animales de traspatio son mayormente ejercidos por las mujeres de la casa (ej. CARDOSO et al., 2015). 
En segundo lugar, las mujeres se distinguen por ser las principales encargadas de la trasmisión y mantenimiento de saberes ligados a plantas y animales, no solo en sus aspectos utilitarios sino también en los simbólicos-cosmológicos. Por ejemplo, estudios en Pilcaniyeu (Provincia de Río Negro), Cuyín Manzano (Provincia de Neuquén), El Escorial y Lagunita Salada (Provincia de Chubut) se evidencia el rol de las mujeres en la enseñanza activa de plantas comestibles y medicinales silvestres y cultivadas (LOZADA; LADIO; WEIGANDT, 2006; EYSSARTIER; LADIO; LOZADA, 2008; RICHERI; CARDOSO; LADIO, 2013; EYSSARTIER; LADIO; LOZADA, 2013). Según Ochoa y Ladio (2015a) en el estudio de plantas con órganos subterráneos comestibles en Cuyín Manzano y Villa LLanquín (Río Negro) se encontró que fueron las madres (52\%) y las abuelas $(26 \%)$ las principales encargadas de la enseñanza, y en menor medida, los padres (16\%) y los abuelos (6\%). En Lozada, Ladio y Weigandt (2004), destacamos que en el proceso de aprendizaje de las personas, el recuerdo de episodios de enfermedad (memoria episódica) está asociado al cuidado de las mujeres en esa situación, y a una acción instructiva por parte de ellas a los integrantes de la familia para que sea aprendido o imitado el uso y/o las formas de recolección de plantas medicinales.

La fuerza de los testimonios tanto de mujeres como hombres en los cuales se mencionan a las madres o abuelas como figuras altamente escuchadas en las comunidades es muy evidente, atraviesan diversas conversaciones y entrevistas. Para señalar algunos, en Igon, Ladio y Lozada (2007), un poblador de Traful (Provincia de Neuquén) dijo "Todos somos buenos conocedores de plantas, pero las mujeres dicen más que los hombres", dando cuenta el rol asignado en la difusión de este tipo de conocimiento. En Ochoa y Ladio (2015b) sobre el uso de los tubérculos comestibles del “yocón” (Diposis patagonica, Apiaceae) encontramos los siguientes testimonios "mi madre jamás se enfermó porque ella solía decir que comía yocón”,y “nosotros somos Mapuche, sabemos y respetamos la tierra..mi abuela me mostró el yocón y por eso yo se lo muestro a mis hijos”. También, en Aigo y Ladio (2016) en la comunidad Mapuche Puel (Provincia del Neuquén) registramos “mi abuela solía decirnos de repente aparecieron estos grandes peces [truchas exóticas, Oncorhynchus mykiss y Salvelinus fontinalis (Salmonidae)], grandes cabezas en el río... [que antes no había] los hombres blancos han estado cerca..." como una mala señal, de advertencia de peligro. Estos testimonios muestran el papel distintivo de la palabra sensible de madres y abuelas, mujeres que son vivenciadas claves de la organización ética de la vida familiar. Son las que diseminan y refuerzan la identidad y las tradiciones locales, 
también suelen ser las voceras de los mensajes que la Naturaleza les brinda para cuidar mejor a su familia y su comunidad (LADIO, 2020b).

En tercer lugar, las mujeres fueron señaladas especialmente como las actoras en el cuidado de salud, hecho que está intrincadamente unido a las formas de obtención de alimentos en respeto con el entorno "solo recolectamos lo que necesitamos" (LADIO; MOLARES, 2017). La alimentación y el cuidado de la salud tienen una connotación sagrada y está basada en las premisas de complementariedad, reciprocidad y equilibrio con todos los elementos de la Naturaleza. Esta premisa ética es la que ellas diseminan y practican desde tiempos ancestrales en pos de lo que hoy llamamos soberanía alimentaria (LADIO; MOLARES, 2017). Es por ello que no solo se ha promovido la inclusión de las mujeres en proyectos de revitalización cultural regional (LADIO, 2006), sino que es explicativo del rol que las mujeres ocupan hoy en día en la lucha por la recuperación de territorios, la denuncia de prácticas nocivas o contaminación medioambiental, a través de la articulación de lo filosófico mapuche y lo político (ALONSO; TRPIN, 2018).

En cuarto lugar, los trabajos muestran que los roles de género en la gestión de los recursos vegetales y animales ligados a la soberanía alimentaria no pueden generalizarse, obedecen a escenarios específicos. Las diferencias entre hombre y mujeres en prácticas y conocimientos encontradas o no en las distintas comunidades y categorías de uso, las hemos entendido no como una resultante inherente al género, de manera esencialista, sino por los distintos contextos u oportunidades de aprendizaje o actuación que cada género accedía según sus circunstancias (LADIO, 2006). Si bien, los trabajos no fueron realizados desde la perspectiva de género, que hubiera podido detectar jerarquías o circunstancias condicionantes para las mujeres, los trabajos han podido identificar contrastes de interés.

Por ejemplo, en Cardoso, Ladio y Lozada (2012) se encontró que los hombres y las mujeres de la comunidad Mapuche Pilkiniyeu del Limay (Provincia de Río Negro) conocen y usan por igual numerosas especies de leñas. Sin embargo, profundizando en las tareas realizadas, vimos que tanto hombres como mujeres recolectan leña de distintos tamaños y lugares. Además, las mujeres, al estar a cargo de mantener el fuego en las cocinas hogareñas, son las principales responsables de la selección pormenorizada de las especies dentro del hogar, según se trate de cocción de alimentos, aromatización de comidas típicas, sahumado de la casa, calefacción u otras necesidades familiares.

En un trabajo con niños y niñas de la comunidad Mapuche Ruka Choroi (Provincia de Neuquén) (CANALE; LADIO, 2020) por medio de dibujos y entrevistas se evidenció 
que las niñas y niños tenían similares conocimiento acerca del pewen (Araucaria araucana, Araucariaceae), la especie arbórea con semillas comestibles más importante y con mayor significación cultural para el pueblo Mapuche. Sin embargo sus formas de recolección parecen ser distintas entre ellos. Un 72\% de las niñas dibujó piñones en el suelo o personas recolectando piñones del suelo describiendo como ellas los recolectan tradicionalmente, mientras que sólo el 12\% de los niños los representó de esta manera. En general, los varones utilizan otras técnicas como el lazo o suben a los árboles para generar el desprendimiento de las semillas de los conos (LADIO; MOLARES, 2017).

En quinto lugar, similarmente a otros lugares del mundo, la horticultura familiar (junto con la ganadería criancera) es base fundamental de la soberanía alimentaria rural patagónica, y es un espacio manejado principalmente por mujeres (CARDOSO et al., 2015). La huerta es el lugar más importante de experimentación y aclimatación de especies y variedades y de enseñanza sobre plantas a los niños de la casa, es el escenario donde se aprende a verlas crecer, a tocarlas, a olerlas y cuidarlas (MOLARES; LADIO, 2017). Son las mujeres quienes conservan las semillas de las plantas que salieron "buenas" para la próxima temporada, elaboran las conservas con los excedentes y son las que comparten semillas y esquejes con otras vecinas, manteniendo así las variedades locales.

Y en sexto y último lugar, los trabajos pudieron señalar la transformación temporal en los roles de género, dando cuenta de procesos dinámicos que acontecen en las comunidades rurales y que repercuten en su soberanía alimentaria. Por ejemplo, un estudio acerca de la práctica de la trashumancia ganadera y la recolección de semillas del pewén $(A$. araucana) entre hombres y mujeres de la comunidad Mapuche Paineo (Neuquén) (LADIO; LOZADA, 2004), resalta que según la literatura, en el pasado ambas tradiciones eran llevadas a cabo por la familia completa. Todos los integrantes participaban de los viajes estableciéndose dos sitios de residencia (la veranada en cordillera de los Andes) y la invernada (tierras más bajas). Sin embargo, en la actualidad esta práctica está en disminución debido a diversas limitaciones territoriales que tienen las comunidades Mapuche. Esta situación es una consecuencia del proceso de despojo que han sufrido las comunidades luego de la Campaña del Desierto a fines del siglo XIX, cuando el estado Argentino en pos de la conformación de su territorio usurpó tierras y fomentó el genocidio de los pueblos originarios patagónicos. Las comunidades quedaron confinadas en sitios cada vez menos productivos, con imposibilidad de trasladarse sin pedir permisos especiales de arreo, sumado a un proceso marcado de emigración de jóvenes hacia áreas urbanas 
(LADIO; LOZADA, 2004). En el presente, la trashumancia la realizan mayormente los varones, por ende ellos tienen mayor oportunidad para explorar los distintos paisajes a lo largo de la ruta de pastoreo y usan mayor cantidad de plantas silvestres comestibles, principalmente originarias de los bosques andino-patagónicos. Mientras que las mujeres, si bien saben en igual medida que los hombres acerca de las plantas comestibles silvestres, ellas restringen su uso a las que se encuentran en los alrededores de la casa y el campo de pastoreo de la invernada dado que deben quedarse en los hogares para que los niños y niñas puedan asistir a la escuela durante el verano.

En síntesis, sugiero que las mujeres rurales Mapuche denotan un amplio margen de acción, y posiblemente esto se deba, en acuerdo con Federici (2010), al sistema de propiedad comunitaria de la tierra, territorio común no solo destinado a la producción, sino también es el escenario donde se despliegan importantes redes de sociabilidad. Y en ese contexto, el rol histórico de las mujeres en esas redes de sociabilidad como cuidadoras y trasmisoras es clave. En consecuencia, del mismo modo que fue señalado por Warren (2009), en nuestros trabajos parece observarse una fuerte construcción de género en el mundo Mapuche que está muy centrada en el papel de las mujeres en el fortalecimiento de la identidad cultural, y por ende se generan numerosas expectativas y sensibilidades entre sus integrantes, y por qué no entre las y los investigadores. Estas expectativas parecieran fomentar un reforzamiento de los mandatos sociales depositados sobre ellas en la soberanía alimentaria. Pero que sin embargo, no hace mayores cuestionamientos en términos de sus derechos.

\subsection{Equívocos patriarcales}

La re-examinación de los trabajos también evidenció algunos equívocos patriarcales que se han puesto de manifiesto en la discursiva y que han operado en la concepción, metodología e interpretación de los resultados. Por ejemplo, la utilización del término sexo equivalente a género es un equívoco patriarcal de un alcance generalizado, especialmente en la literatura más vieja (LADIO; LOZADA, 2004; LADIO, 2006; LOZADA; LADIO; WEIGANDT, 2006). Sin embargo, deseo señalar especialmente otros equívocos que he distinguido de la siguiente manera como: 1) invisibilización, 2) sobre-generalización y 3) simplificación. Los mismos ejemplifican situaciones que sería oportuno no reproducir en futuro, sino como dice Viveros de Castro (2004) y Furlan et al. (2020) que los equívocos sirvan de herramienta de mayor comprensión y reflexión. 


\subsubsection{Invisibilización}

El equívoco de la invisibilización ocurre cuando no hablamos ni de mujeres ni de hombres en nuestros trabajos. En las descripciones de la metodología nos referimos solo al número total de pobladores, se habla de personas, habitantes, unidades familiares, campesinos, familias, informantes sin género, y se describen sus edades promedio. Podemos tomar de ejemplo distintos estudios de caso referidos al uso y conocimiento de plantas silvestres comestibles (LADIO, 2001; LADIO; LOZADA, 2001; LADIO; LOZADA, 2003; CHAMORRO; LADIO, 2020), medicinales (ESTOMBA; LADIO; LOZADA, 2005; OCHOA; LADIO; LOZADA, 2010; MOLARES; LADIO, 2012) у leñateras (RICHERI; CARDOSO; LADIO, 2013). Dichos trabajos han incluido diversas comunidades Mapuche y Mapuche-Tehuelche (Kom Keñi Mu (Río Negro); Rams, Paineo, Cayulef, Painefilu y Curruhuinca (Neuquén); Lago Rosario (Chubut)) y criollas (Cuyín Manzano (Neuquén); Arroyo las Minas (Río Negro); Lagunita Salada y El Escorial (Chubut)). Se describen especies, recetas medicinales, alimentos locales, se habla de tradiciones culinarias, de las prácticas de recolección de distintas especies en el territorio. Sin embargo, en ellos está ausente la referencia sobre las mujeres (y hombres), aun cuando se ha estudiado relaciones vinculadas a la soberanía alimentaria. La discursiva se refiere en todos estos casos a la descripción de un patrimonio biocultural colectivo construido a lo largo del tiempo y arraigado en costumbres de larga data.

Esta invisibilización se puede atribuir a múltiples razones. Una primera y directa es que los objetivos de estos artículos no referían a preguntas referidas al género. Dicha indiferenciación de los participantes fue una manera de mostrar que nuestro diseño de muestreo incluyó a unidades homogéneas y representativas. Una segunda, es que hemos sido poco sensibles y hemos despersonalizado.

Según Vazquez-Garcia (2007) y Ladio (2020a), esto no es atípico, varios trabajos han ignorado la presencia de mujeres (y de hombres) de esta manera. Este equivoco ahora vivenciado desde la perspectiva de género, significa la omisión de las relaciones diversas, complejas y no igualitarias existentes entre hombres y mujeres. Este silenciamiento estaría naturalizando, por ejemplo, que en el interior de las unidades familiares hay mujeres que cocinan, curan, preparan la huerta, trasmiten saberes, buscan leña y otros bienes naturales, sin necesidad ni derecho de ser mencionadas ni ser reconocidas. 


\subsubsection{Sobre-generalización}

Este tipo de equívoco sucede como en el caso de arriba cuando el enfoque del trabajo es a nivel comunitario o de unidades familiares, por ende las individualidades de los participantes no son profundizadas. En este caso, los géneros de los participantes, de mujeres (y de hombres) están explicitados en la metodología de las investigaciones, pero luego este aspecto no es analizado en el trabajo, aunque se evidencien sesgos como resultado del proceso de selección de participantes.

Por ejemplo, en estudios sobre horticultura familiar de la región de la estepa patagónica en las comunidades de Pilcaniyeu, Comallo y Pilkinilley del Limay (Río Negro) (EYSSARTIER; LADIO; LOZADA, 2011a, 2013; 2015) se ha diferenciado el número de participantes femeninos y masculinos. En la metodología se describe que mayoritariamente las mujeres son las entrevistadas dado que se preguntó por la persona encargada de la huerta. Sin embargo, en los resultados cuando se habla de siembra de especies, recolección de plantas, intercambio y guardado de semillas, las mujeres no son mencionadas como las actoras principales, se habla en términos familiares. Por ejemplo, en la comunidad Pichi Leufu (Río Negro), se entrevistó a 16 mujeres y un hombre sobre horticultura familiar (EYSSARTIER; LADIO; LOZADA, 2011b), sin embargo en los resultados no se hace referencia que los conocimientos de 173 especies cultivadas y recolectadas fueron citados mayoritariamente por mujeres.

En Molares y Ladio (2009) se estudió el conocimiento de plantas medicinales en la comunidad Mapuche Lago Rosario (Chubut) en donde participaron 20 mujeres y 6 hombres. Como resultado, se describieron de forma extendida a toda la comunidad las propiedades organolépticas y objetivos medicinales de 75 plantas sanadoras. Del mismo modo, en la comunidad Mapuche Curruhuinca (Neuquén), se trabajó junto con 18 mujeres y 4 hombres, y luego se generalizó el conocimiento de 89 especies (268 usos) a toda la comunidad (ESTOMBA; LADIO; LOZADA 2006).

Es decir, este tipo de equivoco ocurre cuando se toma la información de un grupo y se la generaliza a toda la población. En los estudios arriba señalados se extendieron los saberes principalmente de mujeres a los hombres de la comunidad sintetizando condiciones de género que pueden ser muy diferentes. Esta prolongación de conclusiones válidas para un género como aplicables a ambos, excluye la posibilidad de problematizar sobre la posición de las mujeres en cada uno de los contextos analizados. 


\subsubsection{Simplificación}

Este equivoco sucede cuando en los textos se reduce a la unidad familiar como una unidad nuclear hetero-normativa estándar, hecho que imposibilita mostrar otras diversidades de su interior. Esta simplificación arrasa con la posibilidad de analizar diferencias, roles y posibles jerarquías existentes. Esta simplificación se evidencia cuando, por ejemplo, no se identifican a las mujeres jefas de hogar en los estudios (LADIO, 2020a). O cuando se romantiza la complementariedad entre hombres y mujeres en el trabajo de la agricultura familiar cuando se describen actividades distintas realizadas por ambos géneros en una misma familia. A dichas actividades se las presenta como si fueran igualitarias aun cuando pueden ser muy asimétricas en términos de trabajo (RICHERI; LADIO; BEESKOW, 2013).

Por ejemplo, en las localidades de la Meseta del Chubut (Sierra Rosada, Sierra Colorada y El Escorial), en el estudio del uso de plantas veterinarias, se registró que hombres y mujeres usan un número similar de plantas, interpretándose que las prácticas y saberes del cuidado animal se complementan y comparten entre los integrantes de la familia (CASTILLO; LADIO, 2017). Sin embargo, solo un 25\% de las entrevistas incluyó a mujeres como encargadas de la cría de los animales, hecho que no solo podría estar mostrando su participación minoritaria en la gestión campesina, sino también se omiten las diferentes vicisitudes que las mujeres del campo enfrentan en comparación con los varones.

Otro caso es cuando se convalidan posibles restricciones del uso del espacio para las mujeres, quienes casi siempre son asociadas a los espacios peri domésticos (EYSSARTIER; LADIO; LOZADA, 2011a,b; LADIO; LOZADA, 2004). Una mirada más atenta podría vislumbrar que estos resultados son la consecuencia de roles asignados culturalmente, que limitan el accionar femenino a la casa. Este hecho debe ser complejizado considerando las expectativas y tradiciones propias de cada sociedad (LADIO, 2020a).

\section{REFLEXIONES CONCLUSIVAS}

Los aciertos y los equívocos patriarcales (invisibilización, sobre-generalización y simplificación) mostrados en esta re-evaluación bibliográfica pueden servir de ejemplo para que otros autores y autoras revisen sus posibles sesgos de género. No es fácil clarificar relaciones sociales que no son evidentes sin una perspectiva de género, pero una vez que son ejemplificadas, es posible que podamos dejar de lado estereotipos normalizantes y complejizar el rol de las mujeres rurales. 
La fuerte construcción social existente acerca de las mujeres "cuidadoras" ha atravesado toda la literatura, tanto propia como de otros autores. Si tomamos las palabras de Carol Gilligan (2013, p. 50-51) "En un contexto patriarcal, el cuidado es una ética femenina. Cuidar es lo que hacen las mujeres buenas [dentro de la casa], y las personas que cuidan realizan una labor femenina; están consagradas al prójimo, pendientes de sus deseos y necesidades, atentas a sus preocupaciones; son abnegadas. En un contexto democrático, el cuidado es una ética humana. Cuidar es lo que hacen los seres humanos; cuidar de uno mismo y de los demás es una capacidad humana natural", es necesario que en nuestro trabajo podamos repensar esa construcción de sentido con vistas a generar un proceso de emancipación y reflexión conjunta entre las mujeres rurales y las investigadoras e investigadores.

Redirigir nuestra mirada hacia la interioridad de las mujeres rurales (y de los hombres y sus masculinidades) en la soberanía alimentaria es el camino. Esta revisión además muestra que debe realizarse en clave de interseccionalidad (PENA, 2017), es decir, analizando la convergencia de distintas formas de opresión, debidas al género, pero también vinculadas a la etnicidad y la clase social. Y en este sentido, las mujeres rurales Mapuche en relación a la soberanía alimentaria son intersectadas por diversas formas de desigualdades socioculturales y económicas. Ahondar en estos aspectos donde existen el dolor, la angustia, las frustraciones derivadas de múltiples estructuras de sometimiento y que restringen tanto a varones como a mujeres es fundamental. Además, el despliegue de estrategias y de prácticas igualitarias para sostener la soberanía alimentaria debería ser el nuevo paradigma para la sostenibilidad rural.

\section{AGRADECIMIENTOS}

Un cálido agradecimiento a todas las agricultoras familiares del Noroeste de la Patagonia con quienes he aprendido sobre el amor a la tierra y a todos sus habitantes humanos y no humanos. Especial agradecimiento al Consejo Nacional de Investigaciones Científicas y Técnicas de la Argentina, al INIBIOMA y a la Universidad Nacional del Comahue.

\section{REFERENCIAS BIBLIOGRÁFICAS}

AIGO, J.; LADIO, A. H. Traditional Mapuche ecological knowledge in Patagonia, Argentina: fishes and other living beings inhabiting continental waters, as a reflection of processes of change. Journal of Ethnobiology and Ethnomedicine, v. 12, n. 56, p. 1-17, 
2016.

ALONSO, G.; TRPIN, V. Territorios y cuerpos en el norte de la Patagonia: desafíos teóricos y metodológicos en tiempos de extractivismo. Revista de Estudios Marítimos y Sociales, 2018. Disponible en: https://estudiosmaritimossociales.org/archivo/rems13/dossier-alonso-trpin/. Aceso en: 10 jun. 2020.

BAIN, J. Mexican rural women's knowledge of the environment. Mexican Studies, v. 9, p. 259-274, 1993.

BALDAUF, C. From the colonialist to the " autobotanical " approach : the evolution of the subject-object relationship in ethnobotanical research. Acta Bot. Bras., v. 33, n June, p. 386-390, 2019.

BOURDIEU, P. El sentido práctico. Buenos Aires: Siglo XXI, 2007. 456 p.

BROUCHOUD, S. Desafíos en el abordaje del trabajo rural en el norte de la Patagonia: mujeres en forestación, horticultura y fruticultura. Trabajo y Sociedad, v. 28, p. 267-280, 2017.

BYERS, E.; SAINJU, M. Mountain ecosystems and women: opportunities for sustainable development and conservation. Mountain Research and Development, v.14, n. 213, 1994.

CANALE, A.; LADIO, A. La recolección de piñones de pewen (Araucaria araucana): una situación significativa que conecta a niños mapuches con la naturaleza. Gaia Scientia, 14(1), 12-32, 2020.

CARDOSO, M. B.; LADIO, A. H.; LOZADA, M. The use of firewood in a Mapuche community in a semi-arid region of Patagonia, Argentina. Biomass and Bioenergy, v. 46, n. 0 , p. 155-164, 2012.

CARDOSO, M. B. et al. A. Las mujeres y las plantas.la subsistencia d e las comuniades rurales de la Patagonia árida. LEISA: Revista de Agroecología, v. 31, p. 20-23, 2015.

CASTILLO, L.; LADIO, A. H. Traditional veterinary solutions for herders living in limited and changing conditions: A case study of "crianceros" of Central Northern Patagonia, Argentina. Journal of Arid Environments, v. 145, p. 90-101, 2017.

CHAMORRO M.F; LADIO, A. H. Native and exotic plants with edible fleshy fruits utilized in Patagonia and their role as sources of local functional foods. BMC Complementary Medicine and Therapies, v. 20, n. 155, 2020. Disponible en: https://doi.org/10.1186/s12906-020-02952-1. Aceso en: 10 oct. 2020.

DUARTE ALMADA, E.; OLIVEIRA E SOUZA, M. Quintais. Memória, Resistência e Patrimônio Biocultural. Belo Horizonte: UEMG, 2007. 192 p.

EICHLER, M.; BURKE, M. A. The BIAS FREE Framework. Canadian Journal of Public Health, v. 97, n. 1, 63-68, 2006.

ESTOMBA, D.; LADIO, A. H.; LOZADA, M. Plantas medicinales utilizadas por una 
comunidad Mapuche en las cercanías de Junín de los Andes, Neuquén. Bol Latinoam Caribe Plant Med Aromat, v. 4, n. 6, p. 107-112, 2005.

ESTOMBA, D.; LADIO, A. H.; LOZADA, M. Medicinal wild plant knowledge and gathering patterns in a Mapuche community from North-western Patagonia. Journal of Ethnopharmacology, v. 103, p. 109-119, 2006.

EYSSARTIER, C.; LADIO A. H.; LOZADA, M. Cultural transmission of traditional knowledge in two populations of North-Western Patagonia. Journal of Ethnobiology and Ethnomedicine, v. 4, n.25, p. 1-8, 2008.

EYSSARTIER, C.; LADIO, A. H.; LOZADA, M. Horticultural and gathering practices complement each other: A case study in a rural population of Northwestern Patagonia. Ecology of Food and Nutrition, v. 50, n. 5, p. 429-451, 2011 b.

EYSSARTIER, C.; LADIO A. H.; LOZADA, M. Horticultural practice and germplasm conservation: a case study in a rural population of the Patagonian steppe. Food Security, v. 7, p. 1259-1271, 2015.

EYSSARTIER, C.; LADIO, A. H.; LOZADA, M. Traditional horticultural and gathering practices in two semi-rural populations of Northwestern Patagonia. Journal of Arid Environments, v.97, p. 18-25, 2013.

EYSSARTIER, C.; LADIO, A. H.; LOZADA, M. Traditional horticultural knowledge change in a rural population of the Patagonian steppe. Journal of Arid Environments, v. 75 , n. 1, 78-86, 2011a.

FAO. Efectos de la COVID-19 en hombres $y$ en mujeres y respuestas políticasequitativas en el ámbito de la agricultura, la seguridad alimentaria y la nutrición , 2020. 1-12 p. Disponible en: http:/www.fao.org/3/ca9198es/ca9198es.pdf. Aceso en: 15 oct. 2020.

FEDERICI, S. Calibán y la bruja. Mujeres, Cuerpo y Acumulación Originaria. Madrid, Traficantes de Sueños, 2010. 368 p.

FURLAN, V. et al. Ethnobiological 'Equivocation'and other misunderstandings in the interpretation of natures. Studies in History and Philosophy of Biol \& Biomed Sci. July, 2020. Disponible en: https://doi.org/10.1016/j.shpsc.2020.101333. Aceso en: 17 oct. 2020.

GUILligan, C. La Ética del Cuidado, Barcelona, Cuadernos de la Fundació Víctor Grífols i Lucas, v. 30, 2013. 113 p.

IGON, P; LADIO, A. H.; LOZADA, M. Plantas Medicinales utilizadas en las comunidades de Villa Traful y Cuyín Manzano. Ediciones Imaginaria, Bariloche, 2007 $67 \mathrm{p}$.

KELLEHER, M. The Feminization of Famine: Representations of Women in Famine Naratives. Duke University Press, Nueva York, 1997. 272 p.

LADIO, A. H. A new set of tools for Ethnobiologist in the COVID-19 Pandemic. 
Ethnobiology and Conservation, v. 29, n. July, 2020b. Disponible en: https://doi.org/10.15451/ec2020-07-9.29-1-8. Aceso en: 30 jul. 2020.

LADIO, A. H. Ethnobiology and research on global environmental change: what distinctive contribution can we make? Ethnobiology and Conservation, v. 6, n. 7, 2017. Disponible en: http://doi.org/10.15451/ec2017-07-6.7-1-8. Aceso en: 30 jul. 2020.

LADIO, A. H. Gathering of wild plant foods with medicinal use in a Mapuche community of Northwest Patagonia. In: Pieroni, A.; Price L. L. Eating and Healing: Explorations of Traditional Food as Medicines, USA, Haworth Press, 2006. p. 297-321.

LADIO, A. H. La etnobiología en áreas rurales y su aporte a la lucha para desentrañar sesgos patriarcales. Ethnoscientia, v.5, n.1, p.1-13, 2020a.

LADIO, A. H. The Maintenance of wild edible plant gathering in a Mapuche community of Patagonia. Economic Botany, v. 55, n. 2, p. 243-254, 2001.

LADIO, A. H.; LOZADA, M. Comparison of wild edible plant diversity and foraging strategies in two aboriginal communities of northwestern Patagonia. Biodiversity \& Conservation, v. 12, p. 937-951, 2003.

LADIO, A. H.; LOZADA, M. Non-timber forest product use in two human populations from NW Patagonia: A quantitative approach. Human Ecology, v. 29, n.4, p. 367-380, 2001 .

LADIO, A. H.; LOZADA, M. Summer cattle transhumance and wild edible plant gathering in a Mapuche community of Northwestern Patagonia. Human Ecology, v. 32, p. 225-240, 2004.

LADIO, A. H.; MOLARES, S. Etnoconservacionismo y prácticas locales en Patagonia: avances y perspectivas. In: CASAS, A.; TORRES-GUEVARA, J.; PARRA, F. Domesticación en el Continente Americano. Historia y perspectivas del manejo de recursos genéticos en el Nuevo Mundo, Universidad Agraria La Molina, Lima, Perú, 2017. p. 649-672.

LOZADA, M.; LADIO, A.; WEIGANDT, M. Aspectos de la adquisición y transmisión del conocimiento sobre plantas silvestres en una comunidad rural del Noroeste de la Patagonia. Reunión Internacional Mente y Cultura, Bariloche p. 1-14, 2004.

LOZADA, M.; LADIO, A.; WEIGANDT, M. Cultural transmission of ethnobotanical knowledge in a rural community of northwestern Patagonia, Argentina. Economic Botany, v.60, n. 4, p. 374-385, 2006.

MOLARES, S; LADIO, A. Chemosensory perception and medicinal plants for digestive ailments in a Mapuche community in NW Patagonia, Argentina. Journal of Ethnopharmacology, v. 123, n. 3, p 397-406, 2009.

MOLARES, S.; LADIO, A. Mapuche perceptions and conservation of Andean Nothofagus forests and their medicinal plants: a case study from a rural community in Patagonia, Argentina. Biodiversity and Conservation, v. 21, n. 4, p. 1079-1093, 2012. 
OCHOA, J. J.; LADIO, A. H. Current use of wild plants with edible underground storage organs in a rural population of Patagonia: Between tradition and change. Journal of Ethnobiology and Ethnomedicine, v. 11, n.70, p. 1-14, 2015 a.

OCHOA, J. J.; LADIO, A.H. Plantas silvestres con órganos subterráneos comestibles: transmisión cultural sobre recursos subutilizados en la Patagonia (Argentina). Bol Latinoam Caribe Plant Med Aromat, v. 14, p. 287-300, 2015 b.

OCHOA, J.; LADIO, A.; LOZADA, M. Uso de recursos herbolarios entre mapuches y criollos de la comunidad campesina de Arroyo Las Minas (Río Negro, Patagonia Argentina). Bol Latinoam Caribe Plant Med Aromat, v. 9, n. 4, p. 269-276, 2010.

PENA, M. Hacia una voz propia y feminista en el movimiento campesino de Santiago del Estero. Investigaciones Feministas, v. 8, 2017.

PEREZ, P. Historia y silencio: La conquista del Desierto como genocidio no-narrado. Corpus, Archivos Virtuales de La Alteridad Americana, v. 1, n. 2, 2011. Disponible en: http://ppct.caicyt.gov.ar/index.php/corpus. Aceso en: 09 oct. 2020.

PFEIFFER, J. M.; BUTZ, R. J. Assessing cultural and ecological variation in ethnobiological research: The importance of gender. Journal of Ethnobiology, v. 25, n.2, p. 240-278, 2005.

RICHERI, M.; CARDOSO, M. B.; LADIO, A. H. Soluciones locales y flexibilidad en el conocimiento ecológico tradicional frente a procesos de cambio ambiental: Estudios de caso en Patagonia. Ecología Austral, v. Diciembre, p.184-193, 2013.

RICHERI, M.; LADIO A. H.; BEESKOW, A. M. Conocimiento tradicional y autosuficiencia: la herbolaria rural en la Meseta Central del Chubut (Argentina). Bol Latinoam Caribe Plant Med Aromat, v. 12, n.1, p. 44 - 58, 2013.

SILIPRANDI, E. Mujeres y agroecología. nuevos sujetos políticos en la agricultura familiar. Investigaciones Feministas, v. 1 p. 125-137, 2010.

TASSI, É. M. M.; BEZERRA, I. A Soberania alimentar que desperta e aprofunda os saberes em direitos por terra, por comida de verdade e por igualdade de gênero. Revista Em Extensão, v. Maio, p. 42-52, 2020.

TRPIN, V.; RODRIGUEZ, M. D.; BROUCHOUD, S. Desafíos en el abordaje del trabajo rural en el norte de la Patagonia: Mujeres en forestación, horticultura y fruticultura. Trabajo y Sociedad, v 28, p. 267-280, 2017.

VAZQUEZ-GARCIA, V. Gender, ethnicity, and economic status in plant management: uncultivated edible plants among the Nahuas and Popolucas of Veracruz, Mexico. Agriculture and Human Values, v. 25, p. 65-77, 2007.

VÍA CAMPESINA. ¡Soberanía Alimentaria YA! Una guia por la Soberania Alimentaria, 2018. p. 1-32. Disponible en: https://viacampesina.org/en/wpcontent/uploads/sites/2/2018/02/Food-Sovereignty-a-guide-ES-version-low-res.pdf. Aceso 
en: 30 oct. 2020 .

VIEIRA, B. B.; MILWARD-DE-AZEVEDO, M. A. A etnobotânica e o ecofeminismo em prol da conservação ambiental. Diversidade e Gestão, v. 2, p. 178-188, 2018.

VIVEROS DE CASTRO, E. Perspectival anthropology and the method of controlled equivocation. Tipití, v.2, n. 21, p. 3-22, 2004.

VIZCARRA BORDI, I. Entre las desigualdades de género: un lugar para las mujeres pobres en la seguridad alimentaria y el combate al hambre. Argumentos, v. 21, n. 57, p. 141-173, 2008.

WARREN, S. D. How will we recognize each other as Mapuche?: Gender and ethnic identity performances in Argentina. Gender \& Society, v. 23, n. 6, p.768-789, 2009. 